\title{
A Method for Modeling Noise in PET Images
}

\author{
Manolekshmi.I \\ Assistant Professor, Computer Science and Engineering Baselios Mathews II College of Engineering, \\ Sasthamcotta, Kollam, Kerala - 690520
}

\begin{abstract}
This paper will deal mainly with the performance study and analysis statistical properties of the noise found in various PET images. The method is specifically designed for types of noise produced by acquisition and transmission sequence in PET images. Here signal fluctuations generally originate in the physical processes of imaging rather than in the tissue textures. Various types of noise (transmission and quantization) often contribute to degrade PET images; the overall noise is generally assumed to be additive with a zero-mean, constant-variance Gaussian distribution. However, statistical analysis suggests that the noise variance could be better modeled by a nonlinear function of the image intensity depending on external parameters related to the image acquisition protocol. We present a method to extract the relationship between image intensity and the noise variance and to evaluate the corresponding parameters. The method was applied successfully to PET images with different acquisition sequences and transmission sequence.
\end{abstract}

Keywords: Image processing, Positron emission tomography image, noise measurement.

\section{Introduction}

Image noise is a common problem in most image processing applications as evident in the extensive literature on the ways to reduce or circumvent it. The method is specifically designed for types of noise produced by acquisition and transmission sequence.. Here signal fluctuations generally originate in the physical processes of imaging rather than in the tissue textures. Various types of noise (transmission and quantization) often contribute to degrade PET images; the overall noise is generally assumed to be additive with a zero-mean, constant-variance Gaussian distribution. However, statistical analysis suggests that the noise variance could be better modeled by a nonlinear function of the image intensity depending on external parameters related to the image acquisition protocol. We present a method to extract the relationship between image intensity and the noise variance and to evaluate the corresponding parameters. The method was applied successfully to PET images with different acquisition sequences and transmission sequence. This method relies on the measurement of the relationship between the image intensity I and the noise variance $\sigma_{N}^{2}$. This relationship is of the form

$$
\sigma_{N}^{2}=f\left(I, \alpha_{1}, \alpha_{2}, \alpha_{3}, \ldots\right) \quad(1)
$$

and depends on the noise model whereas the values of the parameters $\alpha_{1}, \alpha_{2}, \alpha_{3}, \ldots$ are determined by the image acquisition protocol. Here we can used a new algorithm named nonlinear noise model with simulated annealing, one of the effective technique for noise optimization. This algorithm is used for optimizing the mean square error by adjusting the $\mathrm{w}$ value. In simulated annealing a fitness function is used to optimize w value. We can obtain high SNR value due to optimization

\section{Noise Model}

In this section, we discuss the statistical properties of common type of noise found in medical imaging (Poisson, and Rician) and derive the relationship (1) for each of them. Whenever an image contains different types of uncorrelated noise, the overall noise variance $\sigma_{N}^{2}$ can be expressed by assuming up the various noise contributions

$$
\sigma_{N}^{2}=\sigma_{1}^{2}+\sigma_{2}^{2}+\ldots+\sigma_{n}^{2}
$$

For example, the images from a charge coupled device (CCD) camera are free of grain noise but are degraded by Poisson and read-out noises. The noise variance on such images is either constant or linear dependent on the signal intensity. Unless otherwise mentioned, the images analyzed in this work were such that the noise contributions from secondary sources were negligible.

\section{A. Poisson Noise}

Poisson noise prevails in situations where an image is created by the accumulation of photons over a detector. Typical examples are found in standard X-ray films, CCD cameras, PET images and infrared photometers. We focus our attention on images saved with linear or logarithmic intensity scaling. 


\section{B. Rician Noise:}

The noise in MR images has a Rician PDF. For these tests, we have used a standard volume coil (birdcage) which has uniform efficiency throughout the volume of interest. The signals are acquired in quadrature. Each signal produce an image that is degraded by a zero-mean Gaussian noise of standard deviation (which we define as the noise level). The signals are acquired in quadrature. Each signal produces an image $\mathrm{x}$ that is degraded by a zero-mean Gaussian noise of standard deviation $\sigma_{0}$ (which we define as the noise level). The two images are then combined into a magnitude image I and the Gaussian noise PDF is transformed into a Rician noise PDF. The expectation values for the mean magnitudeand the variance are [2]

$$
\boldsymbol{N}_{i, j}=\bar{I}_{i, j}-\overline{\boldsymbol{I}}_{i, j}
$$

Radionuclides used in PET scanning are typically isotopes with short half-lives such as carbon11 ( 20 min), nitrogen-13 ( 10 min), oxygen-15 ( 2 min), fluorine-18 ( 110 min)., or rubidium-82( 1.27 min). These radionuclides are incorporated either into compounds normally used by the body such as glucose (or glucose analogues), water, or ammonia, or into molecules that bind to receptors or other sites of drug action. Such labelled compounds are known as radiotracers. PET technology can be used to trace the biologic pathway of any compound in living humans (and many other species as well), provided it can be radio labeled with a PET isotope. Thus, the specific processes that can be probed with PET are virtually limitless, and radiotracers for new target molecules and processes are continuing to be synthesized; as of this writing there are already dozens in clinical use and hundreds applied in research. At present, however, by far the most commonly used radiotracer in clinical PET scanning is fluorodeoxy glucose (also called FDG or fludeoxy glucose), an analogue of glucose that is labeled with fluorine-18. This radiotracer is used in essentially all scans for oncology and most scans in neurology, and thus makes up the large majority of all of the radiotracer (> 95\%) used in PET and PETCT scanning.Due to the short half-lives of most positron-emitting radioisotopes, the radiotracers have traditionally been produced using a cyclotron in close proximity to the PET imaging facility. The half-life of fluorine-18 is long enough that radiotracers labeled with fluorine-18 can be manufactured commercially at offsite locations and shipped to imaging centers. Recently rubidium- 82 generators have become commercially available. These contain strontium-82, which decays by electron capture to produce positron-emitting rubidium82

The raw data collected by a PET scanner are a list of 'coincidence events' representing nearsimultaneous detection (typically, within a window of 6 to 12 nanoseconds of each other) of annihilation photons by a pair of detectors. Each coincidence event represents a line in space connecting the two detectors along which the positron emission occurred (i.e., the line of response (LOR)). Modern systems with a higher time resolution (roughly 3 nanoseconds) also use a technique (called "Time-of-flight") where they more precisely decide the difference in time between the detection of the two photons and can thus localize the point of origin of the annihilation event between the two detectors to within $10 \mathrm{~cm}$.

Coincidence events can be grouped into projection images, called sinograms. The sinograms are sorted by the angle of each view and tilt (for 3D images). The sinogram images are analogous to the projections captured by computed tomography (CT) scanners, and can be reconstructed in a similar way. However, the statistics of the data are much worse than those obtained through transmission tomography. A normal PET data set has millions of counts for the whole acquisition, while the CT can reach a few billion counts. This contributes to PET images appearing "noisier" than CT. Two major sources of noise in PET are scatter (a detected pair of photons, at least one of which was deflected from its original path by interaction with matter in the field of view, leading to the pair being assigned to an incorrect LOR) and random events (photons originating from two different annihilation events but incorrectly recorded as a coincidence pair because their arrival at their respective detectors occurred within a coincidence timing window).

PET imaging is an established translational research tool impacting clinical diagnostics and therapeutic recourse for over 2 decades. Redefining and innovative technologies in preclinical PET offer researchers unparalleled access, ultra high sensitivity at a footprint that can be supported by core laboratories or independent labs with access to small animal vivariums

The multimodal platform redefines pre-clinical expectations offering a purpose-built technology for quantitative results enabling more data per animal, minimizing preclinical imaging costs and increase translational impact. The G-Platform is offered in a multimodal suite of PET/X-Ray (G4) or PET/CT (G8) combinations.

\section{Materials And Methods}

The first part of this section describes a method for measuring the relationship (1) between the intensity and the noise variance in an image. A noise image is first generated as the difference between the original image and a smoothed version of it.A mask image is then created to identify the pixels on the imagelplateaus. The noise variance on these image plateaus is then evaluated using robust estimators. The second part describes the 
imaging protocol for the acquisition of $\mathrm{MR}$ and $\mathrm{X}$-ray images. A technique much like the reconstruction of computed tomography (CT) and single-photon emission computed tomography (SPECT) data is more commonly used, although the data set collected in PET is much poorer than CT, so reconstruction techniques are more difficult (see Image reconstruction of PET). Using statistics collected from tens of thousands of coincidence events, a set of simultaneous equations for the total activity of each parcel of tissue along many LORs can be solved by a number of techniques, and, thus, a map of radioactivities as a function of location for parcels or bits of tissue (also called voxels) can be constructed and plotted. The resulting map shows the tissues in which the molecular tracer has become concentrated, and can be interpreted by a nuclear medicine physician or radiologist in the context of the patient's diagnosis and treatment plan.

\section{A. Noise Characterization}

1) Image Smoothing:The smoothing method works well for pixels located on plateaus where the intensity gradients are small. Near the edges, where the intensity gradients are large, the image smoothing does not reproduce the local mean intensity well and the noise signal has a nonzero mean.The filter size depends on image resolution and is found by trial and error. If is too small, the smoothed image tends to follow the original image too closely and the noise variance is underestimated. If is too large, the intrinsic variations in the image are smoothed out and the noise variance is overestimated or may not be of the form (1). All the images in this study were processed with pixels and the limited size of the filter was taken into account by multiplying the noise variance with a correction factor (see Section III).

2) Binary Mask Generation: Edge pixels are discarded in the analysis and are masked out using a binary mask based on the edge information. The mask is first created by applying a threshold to a gradient image computed using Sobel filters. The threshold value is found using a method described in Section IV.The binary mask is eroded by half the size of the smoothing filter to remove the pixels where the computed local mean intensity is imprecise due to the proximity to the image edges. The image boundaries are also eroded to the same depth to remove boundary effects due to filtering. Finally, the binary mask is cleaned from binary noise using standard morphological operators (opening and closing). This method eliminates highly textured regions from the noise analysis.

The first algorithm iteratively minimizes a penalized maximum-likelihood (PML) objective function. It is based on standard de-coupled surrogate functions for the ML objective function and de-coupled surrogate functions for a certain class of penalty functions. As desired, the PML algorithm guarantees nonnegative estimates and monotonically decreases the PML objective function with increasing iterations. The second algorithm is based on an iteration dependent, de-coupled penalty function that introduces smoothing while preserving edges. For the purpose of making comparisons, the MLEM algorithm and a penalized weighted leastsquares algorithm were implemented. In experiments using synthetic data and real phantom data, it was found that, for a fixed level of background noise, the contrast in the images produced by the proposed algorithms was the most accurate.

In the image reconstruction by means of an anlatycal method such as a convolution back projection method it is recognized that the linear sampling interval should be smaller than or atleast equal to the half the spatial resolution to be obtained.The sampling requirement arises from the use of an analytical reconstruction method.The sampling requirement relaxed and may realized the full use of detector.

\section{References}

[1]. S. I. Olsen, "Noise variance estimation in images," presented at the 8thScandinavian Conference on Image Analysis, Tromsø, Norway, May 25-28, 1993.

[2]. B. Waegli, "Investigations into the noise characteristics of digitizedaerial images, Proc. ISPRS Commission II Symp., Cambridge, U.K.July 13-17, 1998,” Int. Arch. Photogrammetry and Remote Sensing,vol. XXXII/2, pp. 341-348.

[3]. H. Lu, X. Li, I. T. Hsiao, and Z. Liang, "Analytical noise treatment for low-dose CT projection data by penalized weighted leastsquaressmoothing in the K-L domain,” Proc. SPIE, Medical Imaging 2002, vol.4682, pp. 146-152.

[4]. H. Gudbjartsson and S. Patz, "The rician distribution of noisy MRI data," Magn. Reson. Med., vol. 34, pp. 910-914, Dec. 1995.

[5]. T. Lei and W. Sewchand, "Statistical approach to X-ray CT imaging an its applications in image analysis-Part I: Statistical analysis of X-ray CT imaging," IEEE Trans. Med. Imag., vol. 11, Apr. 1992.

[6]. J. C. Dainty and R. Shaw, Image Science. New York: Academic, 1974. 